\title{
Evaluation of postmortem measurement of NT-proBNP as a marker for cardiac function
}

\author{
Katarzyna Michaud • Marc Augsburger • \\ Nicolas Donzé • Sara Sabatasso • Mohamed Faouzi • \\ Marc Bollmann • Patrice Mangin
}

Received: 19 December 2007 / Accepted: 20 May 2008/ Published online: 14 June 2008

(C) Springer-Verlag 2008

\begin{abstract}
Clinical biomarkers of cardiac function could also be monitored postmortem. Among the natriuretic peptides, the aminoterminal portion of pro-brain natriuretic peptide (NT-proBNP) appears to be a more reliable postmortem tool than the BNP, owing to its longer half-life and greater stability. In living persons, NT-proBNP is considered to be a marker of heart failure, and its level rises after cardiac ischemia. The goal of this study was first to evaluate the postmortem stability of NT-proBNP, then to measure the NTproBNP levels in postmortem cases of heart failure related to coronary ischemia. The goal of this study was also to evaluate the correlations between different specimens collected at autopsy (e.g. blood, serum, vitreous humor and pericardial fluid). The study included 96 cases, which were classified into 4 groups according to the autopsy and histological findings. The NT-proBNP levels were significantly higher in individuals who had suffered from chronic cardiac ischemia, with or without acute coronary events, than in either control cases or those who had suffered from acute
\end{abstract}

K. Michaud $(\bowtie) \cdot$ M. Augsburger $\cdot$ S. Sabatasso $\cdot$ M. Bollmann $\cdot$ P. Mangin

Centre Universitaire romand de Médecine Légale,

Centre Hospitalier Universitaire Vaudois, University of Lausanne,

Bugnon 21,

1005 Lausanne, Switzerland

e-mail: katarzyna.michaud@chuv.ch

\section{N. Donzé}

Institut Cantonal des Hôpitaux Valaisans,

Grand Champsec 86,

1950 Sion, Switzerland

\section{Faouzi}

Centre d'épidémiologie clinique,

Centre Hospitalier Universitaire Vaudois, University of Lausanne,

Bugnon 17,

1005 Lausanne, Switzerland thromboembolism or acute rupture of a plaque without chronic cardiac ischemia. The highest levels were registered in individuals who had suffered from acute coronary thromboembolism in association with chronic coronary ischemia. Good correlations in the NT-proBNP levels for the different specimens were observed between samples of femoral blood, serum, and pericardial fluid. Our data indicated that postmortem measurements of NT-proBNP are reliable and compatible with clinical findings.

Keywords NT-proBNP - Natriuretic peptides · Heart failure · Coronary ischemia $\cdot$ Forensic autopsy

\section{Introduction}

Acute heart failure is the most common cause of hospitalization and death in Europe, especially in elderly individuals [1]. In most clinical cases, heart failure is a consequence of cardiovascular disease. However, in medicolegal situations, heart failure may be attributable to other causes, and its identification could have important legal implications [2-8]. It could be associated with acute disease or trauma, with drug intoxication or a protracted state of agony.

In clinical medicine, acute heart failure is diagnosed primarily on the basis of clinical symptoms. Nevertheless, biochemical markers are very useful tools in diagnosis as well as in assessing risk factors and therapeutic decision making [911]. In forensic medicine, the diagnosis of heart failure is more difficult. It is impossible to detect the usual clinical symptoms, and in many cases, the autopsy findings are nonspecific for the diagnosis of heart failure. The usual postmortem signs of heart failure include cardiac dilatation and visceral congestion of the internal organs. But, these features are difficult to assess objectively, especially after a long postmortem period. 
Clinical biochemical markers of cardiac function have also been monitored postmortem. However, for many markers, such as troponin, the postmortem analyses are unreliable or controversial $[12,13]$. The natriuretic peptides, especially the aminoterminal portion of pro-brain natriuretic peptide (NT-proBNP), have recently been introduced as biomarkers of cardiac function. BNP and NTproBNP are neurohormones, synthesized primarily by atrial or ventricular myocardial cells. These neurohormones are released from cardiac myocytes in response to ventricular wall stress, ischemia, or infarction. The biological effects of BNP include diuresis, vasodilatation, and inhibition of rennin and aldosteron production and of cardiac and vascular myocytes growth. Whether NT-proBNP has biological effects on its own is currently unknown [14]. BNP and NT-proBNP differ in their biochemical and physiological characteristics [15-17]. BNP is hormonally active, with a half-life of approximately $22 \mathrm{~min}$, whereas NT-proBNP, the inactive precursor of BNP, lacks a clearance receptor and, therefore, has a longer half-life (about $120 \mathrm{~min}$ ). The circulating concentration of NTproBNP is believed to be less susceptible to the condition under which the blood sample is taken [18]. In addition, NT-proBNP appears to be more discerning marker of early cardiac dysfunction than BNP $[16,19]$. The goal of this preliminary study was to evaluate the possibility of measuring NT-proBNP postmortem. To this end, we evaluated first the postmortem stability of NT-proBNP. Then, in order to control the correlation with clinical observation, we monitored the NT-proBNP levels in cases of sudden cardiac death which were clinically attributed to heart failure from coronary ischemia and in control cases without cardiac pathology. The last goal of this study was to compare the correlations of levels of NT-proBNP within different specimens (blood, serum, vitreous humor, and pericardial fluid) that were collected during the autopsies.

\section{Materials and methods}

A total of 96 cases ( 73 males and 23 females) were selected from forensic autopsies that had been performed between 2002 and 2006. The age of the persons ranged from 31 to
90 years at death. The estimated postmortem period did not exceed $24 \mathrm{~h}$. In each case, the autopsy was followed by a histological examination. Cases of liver or kidney failure were excluded from the study. Examination of the heart had involved macroscopical inspection and a histological analysis of myocardial specimens that had been derived from at least five different topographic locations, namely left anterior, left lateral, left posterior, septal, and right lateral. The sections were routinely stained with hematoxyline-eosin. In 56 cases, signs of acute or chronic cardiac ischemia were identified. In the cases of acute cardiac ischemia, there was evidence of either acute coronary thromboembolism or acute plaque rupture. The criteria for chronic ischaemic heart disease included pathology of the coronary artery with a significant degree of atherosclerotic stenosis (more than 75\%) and histopathologic changes in myocardium (e.g., fibrotic foci). The 40 control cases included deaths due to noncardiac causes with neither macroscopical nor microscopical signs of coronary ischemia.

According to the medical records and the autopsy findings, the cases were classified into four groups:

Group A: Acute coronary thromboembolism or acute plaque rupture without evidence of chronic cardiac ischemia $(n=18)$,

Group B: Acute coronary thromboembolism or acute plaque rupture in association with chronic coronary ischemia $(n=13)$,

Group C: Chronic cardiac ischemia without acute coronary events $(n=25)$,

Group D: Control cases, attributed to noncardiac causes with no signs of cardiac ischemia $(n=40)$.

Ages and sex of cases are detailed in Table 1.

In groups $\mathrm{A}$ and $\mathrm{B}$, the cause of death was attributable to coronary ischemia, in group C, 17 deaths (68\%) were attributable to coronary ischemia, two to gunshot lesions, three to trauma, two to carbon monoxide intoxication, and one to stabbing.

In the control group, the causes of death were gunshot lesions in 16 cases, trauma in 18 , and asphyxia in six (hanging in five cases and drowning in one).

Femoral blood, serum, vitreous humor and pericardial fluid were collected during the autopsy. Serum was derived

Table 1 Levels of NT-proBNP [mean value \pm SD (pg/mL)]

\begin{tabular}{|c|c|c|c|c|c|c|c|c|c|}
\hline Groups & Number & $\begin{array}{l}\text { Male/ } \\
\text { female }\end{array}$ & $\begin{array}{l}\text { Age } \\
\text { range }\end{array}$ & $\begin{array}{l}\text { Age } \\
\text { (years) }\end{array}$ & $\begin{array}{l}\text { Heart } \\
\text { weight }\end{array}$ & Serum & $\begin{array}{l}\text { Femoral } \\
\text { blood }\end{array}$ & $\begin{array}{l}\text { Vitreous } \\
\text { fluid }\end{array}$ & $\begin{array}{l}\text { Pericardial } \\
\text { fluid }\end{array}$ \\
\hline A (acute coronary ischemia) & 18 & $18 / 0$ & $31-59$ & $48 \pm 10$ & $431 \pm 65$ & $108 \pm 156$ & $28 \pm 36$ & $183 \pm 138$ & $1756 \pm 1718$ \\
\hline $\begin{array}{l}\text { B (acute and chronic coronary } \\
\text { ischemia) }\end{array}$ & 13 & $9 / 4$ & $47-86$ & $62 \pm 13$ & $439 \pm 88$ & $688 \pm 309$ & $536 \pm 310$ & $323 \pm 217$ & $18070 \pm 12596$ \\
\hline C (chronic coronary ischemia) & 25 & $20 / 5$ & $33-90$ & $62 \pm 14$ & $486 \pm 144$ & $310 \pm 294$ & $238 \pm 198$ & $288 \pm 301$ & $5441 \pm 4408$ \\
\hline D (control) & 40 & $30 / 10$ & $18-72$ & $39 \pm 14$ & $344 \pm 81$ & $68 \pm 64$ & $33 \pm 30$ & $168 \pm 147$ & $1236 \pm 1492$ \\
\hline
\end{tabular}


from a blood sample, which was centrifuged immediately after collection. Postmortem specimens were stored at $-20^{\circ} \mathrm{C}$ until the time of analysis.

To evaluate the stability of NT-proBNP in postmortem blood, one sample was divided into three groups of five aliquots, which were analyzed on days $0,3,9,15$, and 24 . The first group of aliquots was maintained at ambient temperature, the second group at $+4^{\circ} \mathrm{C}$, and the third group at $-20^{\circ} \mathrm{C}$. A similar experimental set-up was established for the samples of pericardial fluid sample from the same case.

The concentration of NT-proBNP was measured using a chemiluminescent immunoassay kit (Elecsys 2010 analyser, Roche Diagnostics, Basel, Switzerland; Dimension Xpand plus, Dade Behring, Deerfield, IL, USA).

The data obtained from groups A-D were statistically analyzed using nonparametric Mann-Whitney test. The correlations between sets of data were evaluated using Spearman's correlation test.

\section{Results}

Stability of NT-proBNP within postmortem samples of blood and pericardial fluid

Data pertaining to the stability of NT-proBNP within postmortem samples of blood and pericardial fluid that had been stored at different temperature for $24 \mathrm{~h}$ are presented in Fig. 1. At ambient temperature, NT-proBNP underwent substantial degradation within both body fluids during the course of 24 days (50\% in blood and $37 \%$ in pericardial fluid). Within blood samples, NT-proBNP was less prone to degradation at $-20^{\circ} \mathrm{C}$ than at $4^{\circ} \mathrm{C}$, but within samples of pericardial fluid, the rate of degradation of NTproBNP was similar at $-20^{\circ} \mathrm{C}$ and at $4^{\circ} \mathrm{C}$. After 24 days, only $16 \%$ of the NT-proBNP had been degraded.

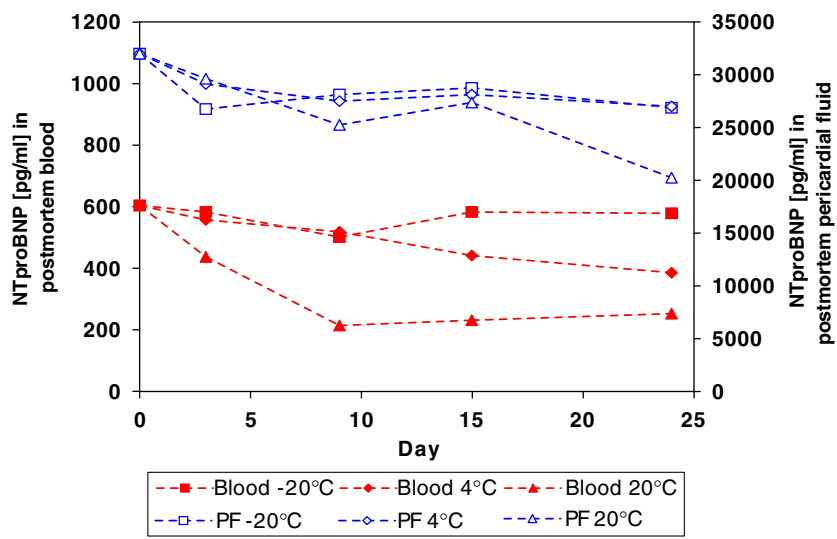

Fig. 1 Stability of NT-proBNP within postmortem samples of blood and pericardial fluid
NT-proBNP levels in the different groups and bodily fluids

Within samples of blood, serum, and pericardial fluid, the levels of NT-proBNP were significantly higher $(p<0.001)$ in individuals who had suffered from chronic cardiac ischemia, with or without acute coronary events (groups B and C), than in either control persons (group D) or those who had suffered from acute coronary thromboembolism or acute rupturing of a plaque without chronic cardiac ischemia (group A).

The highest values were registered in individuals who had suffered from acute coronary thromboembolism in association with chronic coronary ischemia (group B). Within samples of the vitreous humor, the differences between the groups were not significant ( $p>0.05$; Fig. 2, Tables 1 and 2).

The most striking correlations in the levels of NT-proBNP for the different groups were observed between blood and pericardial fluid $(r=0.73)$, between blood and serum $(r=$ $0.77)$, and between serum and pericardial fluid $(r=0.65)$. The correlations between vitreous humor and blood $(r=0.17)$, between vitreous humor and serum $(r=0.11)$, and between vitreous humor and pericardial fluid $(r=0.21)$ were weak.

Dependency of NT-proBNP levels on gender, age and heart weight

To ascertain whether the levels of NT-proBNP within the different bodily fluids were linked with gender, age, and heart weight, a logistic regression model was applied. The odds ratios and $p$ values are presented in Table 3 . In the multivariable analysis for blood samples, only age (odds ratio $=1.006 ; p=0.036$ ) and heart weight (odds ratio $=1.065$; $p=0.002$ ) were significantly associated with higher levels of NT-proBNP.

The logistic regression analysis revealed that blood levels of NT-proBNP rose significantly with an increase in heart weight (odds ratio $=1.00 ; p=0.004$ ) and with an increase in age (odds ratio $=1.072 ; p<0.0001$ ). Similar results were obtained for the levels of NT-proBNP within the serum, pericardial fluid, and vitreous humor. Only within the vitreous humor samples were the levels of NTproBNP significantly different between males and females (odds ratio $=4.54 ; p=0.007$ ).

The age distribution between the four groups differed significantly, except between groups A and D $(p=0.105)$. The heart weight distribution between the four groups likewise differed significantly $(p<0.001)$.

\section{Discussion}

The postmortem levels of natriuretic peptides have been measured in only a few studies. Zhu et al. [20] attributed 

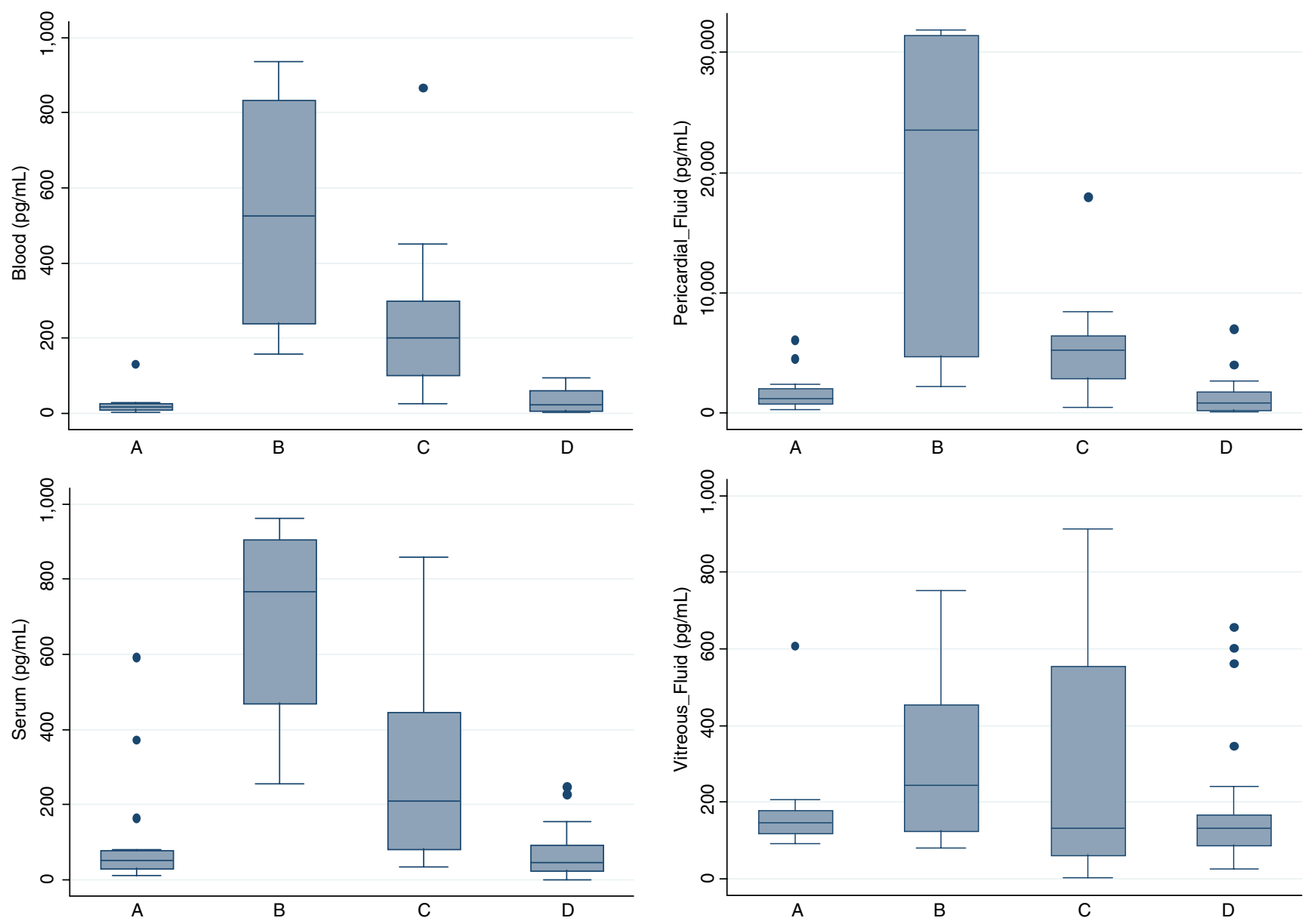

Fig. 2 NT-proBNP levels within different bodily fluids (blood, serum, pericardial fluid, and vitreous humor)

elevation in the postmortem pericardial levels of the atrial natriuretic peptide and the BNP to acute atrial overload and subacute or chronic heart failure, respectively. However, in our opinion, BNP is not suitable for forensic practice. In our experience, BNP cannot be detected in frozen postmortem samples (data not presented). This is not surprising since Mueller et al. have indeed questioned the validity of endogenous BNP measurement in frozen samples [21]. On the basis of our own experience with BNP, and in accordance with published data, we conjectured that for autopsic purposes, NT-proBNP might be a more reliable biomarker than BNP, owing to its greater stability and longer half-life reported in the literature [17, 22-24].

Plasma biomarkers can be very useful diagnostic tools if measurement is reliable. Hence, it is necessary to ascertain whether NT-proBNP decays appreciably during the storage

Table 2 Results of the inter-group analysis using Wilcoxon's two-sample, rank-sum (Mann Whitney) test

\begin{tabular}{llllll}
\hline Groups & & Blood & Serum & Pericardial fluid & Vitreous fluid \\
\hline A & B & $<0.0001(\mathrm{~s})$ & $<0.0001(\mathrm{~s})$ & $0.0005(\mathrm{~s})$ & $0.0647(\mathrm{~ns})$ \\
A & B + C & $<0.0001(\mathrm{~s})$ & $<0.0001(\mathrm{~s})$ & $0.0002(\mathrm{~s})$ & $0.3703(\mathrm{~ns})$ \\
A & D & $0.7967(\mathrm{~ns})$ & $0.9029(\mathrm{~ns})$ & $0.1303(\mathrm{~ns})$ & $0.2644(\mathrm{~ns})$ \\
B + C & D & $<0.0001(\mathrm{~s})$ & $<0.0001(\mathrm{~s})$ & $<0.0001(\mathrm{~s})$ & $0.0385(\mathrm{~s})$ \\
B & C & $0.0031(\mathrm{~s})$ & $0.0021(\mathrm{~s})$ & $0.0406(\mathrm{~s})$ & $0.2758(\mathrm{~ns})$ \\
A + B + C & D & $<0.0001(\mathrm{~s})$ & $0.0005(\mathrm{~s})$ & $<0.0001(\mathrm{~s})$ & $0.0370(\mathrm{~s})$ \\
\hline
\end{tabular}

$s$ Significant, $n s$ nonsignificant 
Table 3 Results of the logistic regression analysis

\begin{tabular}{llcc}
\hline Outcome & Indep-var & Odds ratio & $P$ value \\
\hline Blood & Sex & 0.49 & 0.23 \\
Blood & Age & 1.072 & $<0.001$ \\
Blood & Heart weight & 1.007 & $<0.001$ \\
Serum & Sex & 0.47 & 0.17 \\
Serum & Age & 1.06 & 0.004 \\
Serum & Heart weight & 1.004 & 0.049 \\
Pericardial Fluid & Age & 1.10 & $<0.001$ \\
Pericardial Fluid & Sex & 0.41 & 0.14 \\
Pericardial Fluid & Heart weight & 1.005 & 0.058 \\
Vitreous Fluid & Age & 1.002 & 0.89 \\
Vitreous Fluid & Sex & 4.55 & 0.007 \\
Vitreous Fluid & Heart weight & 1.007 & 0.008 \\
& & & \\
\hline
\end{tabular}

of postmortem samples. Using clinical samples of serum and plasma, Nowatzke et al. demonstrated that NT-proBNP remains stable for at least 1 year at sub-zero temperature [24]. Our own data partially support these findings. After 24 days of storage at $-20^{\circ} \mathrm{C}$, the concentration of NTproBNP in samples of pericardial fluid decreased by no more than $16 \%$. In clinical labolatory practice, only serum NT-proBNP levels are measurable. In forensic practice, in many cases, serum might be unavailable especially in cases of trauma or in decomposed bodies. Therefore, it was important to establish the correlations between serum and other body fluids. In this study, good correlations in the NTproBNP levels for the different groups were observed between samples of serum, blood, and pericardial fluid. Hence, any of these body fluids could be used for postmortem testing. The levels of NT-proBNP in samples of the vitreous humor did not correlate well with those in the other body fluids. A possible explanation for this discrepancy may lie in the existence of the blood-aqueous barrier [25, 26].

In this study, individuals who had suffered chronic cardiac ischemia, with or without acute coronary events (group B and C), had significantly higher levels of NTproBNP than either control persons (group D) or those who had suffered from acute coronary thromboembolism or acute rupturing of plaques without chronic cardiac ischemia (group A). The highest levels were registered in individuals who had suffered from acute coronary thromboembolism in association with chronic cardiac ischemia (group B). According to the literature, in patients with an acute coronary syndrome, the levels of NT-proBNP rise rapidly, with an initial peak after $12-48 \mathrm{~h}$ and a second one after 58 days [27].

The lower levels of NT-proBNP in cases of acute coronary thromboembolism (group A) could be accounted for by the short time elapsing between the formation of the thrombus and death, which intervened before the first peak in NT-proBNP had been attained. As mentioned, individuals who had suffered from acute coronary thromboembolism in association with chronic coronary ischemia (group B) had the highest levels of NT-proBNP. This finding suggests that the existence of chronic coronary ischemia promotes a more rapid and augmented release of NTproBNP in the event of an acute coronary thromboembolism. The augmented releases of NT-proBNP may reflect an increase in gene expression, as suggested for BNP [28]. In clinical practice, high levels of NT-proBNP, which are associated with cardiac diseases in general and with acute coronary syndromes and heart failure in particular, have a poor prognostic value [11, 27, 29-32]. Our own data accord with clinical observations.

In this study, the higher levels of NT-proBNP were correlated to an increase in heart weight. This is not surprising, as heart failure may be associated with an increase in heart weight. The correlation between increase of NT-proBNP levels with an increase in age is compatible with published clinical studies.

In conclusion, the accordance between clinical observations, histopathological findings, and postmortem levels of NT-proBNP within femoral blood, serum, and pericardial fluid indicate that this biomarker of cardiac function could be of diagnostic value in forensic medicine, albeit that it is not helpful in detecting early myocardial ischemia. The postmortem measurement of NT-proBNP could be serviceable in many medicolegal situations, not only in those in which cardiovascular pathology is implicated.

\section{Study limitations}

The number of cases included in this study was fairly small. Nevertheless, our preliminary data are so encouraging as to justify further postmortem investigations of a similar kind with larger cohorts. 
Acknowledgments We are grateful to Anna Arnold, Angélique Chevalley, Magali Dovat Sabatella, Cinzia Forney, Catherine Meylan Bohnenblust, and Joël Stauber for skillful technical assistance with NT-proBNP measurements.

\section{References}

1. Cleland JGF, Swedberg K, Follath F et al (2003) The EuroHeart failure survey programme - a survey on the quality of care among patients with heart failure in Europe: part 1: patient characteristics and diagnosis. Eur Heart J 24:442-463

2. Fineschi V, Karch S, D'Errico S, Pomara C, Riezzo I, Turillazzi E (2006) Cardiac pathology in death from electrocution. Int J Legal Med 120:79-82

3. Fineschi V, Riezzo I, Centini F, Silingardi E, Licata M, Beduschi G, Karch S (2007) Sudden cardiac death during anabolic steroid abuse: morphologic and toxicologic findings in two fatal cases of bodybuilders. Int J Legal Med 121:48-53

4. Hamilton RA, Sullivan L, Wolf BC (2007) Sudden cardiac death due to giant cell inflammatory processes*. J Forensic Sci 52:943-948

5. Pfeiffer H, Al Khaddam M, Brinkmann B, Köhler H, Beike J (2006) Sudden death after isobutane sniffing: a report of two forensic cases. Int J Legal Med 120:168-173

6. Thierauf A, Dettmeyer R, Wollersen H, Madea B (2007) Aplastic right coronary artery and left coronary artery with a separate origin of the circumflex branch in a 31-year-old woman. Forensic Sci Int 173:178-181

7. Wedekind H, Bajanowski T, Friederich P et al (2006) Sudden infant death syndrome and long QT syndrome: an epidemiological and genetic study. Int J Legal Med 120:129-137

8. Wedekind H, Schulze-Bahr E, Debus V, Breithardt G, Brinkmann B, Bajanowski T (2007) Cardiac arrhythmias and sudden death in infancy: implication for the medicolegal investigation. Int J Legal Med 121:245-257

9. Paulus WJ, Tschope C, Sanderson JE et al (2007) How to diagnose diastolic heart failure: a consensus statement on the diagnosis of heart failure with normal left ventricular ejection fraction by the Heart Failure and Echocardiography Associations of the European Society of Cardiology. Eur Heart J in Press

10. Metra M, Nodari S, Parrinello G et al (2007) The role of plasma biomarkers in acute heart failure. Serial changes and independent prognostic value of NT-proBNP and cardiac troponin-T. Eur J Heart Failure 9:776-786

11. Writing Group $M$ et al (2008) National academy of clinical biochemistry laboratory medicine practice guidelines: clinical utilization of cardiac biomarker testing in heart failure. Clin Biochem 41:210-221

12. Davies SJ, Gaze DC, Collinson PO (2005) Investigation of cardiac troponins in postmortem subjects: comparing antemortem and postmortem levels. Am J Forensic Med Pathol 26:213-215

13. Zhu B-L, Ishikawa T, Michiue T et al (2006) Postmortem cardiac troponin $\mathrm{T}$ levels in the blood and pericardial fluid. Part 1. Analysis with special regard to traumatic causes of death. Legal Med 8:86-93

14. Hall C (2004) Essential biochemistry and physiology of (NT-pro) BNP. Eur J Heart Fail 6:257-260

15. Melanson SF, Tanasijevic MJ (2005) Laboratory diagnosis of acute myocardial injury. Cardiovas Pathol 14:56-161
16. Mueller T, Gegenhuber A, Poelz W, Haltmayer M (2004) Headto-head comparison of the diagnostic utility of BNP and NTproBNP in symptomatic and asymptomatic structural heart disease. Clin Chim Acta 341(1-2):41-48

17. Pfister R, Scholz M, Wielckens K, Erdmann E, Schneider CA (2004) Use of NT-proBNP in routine testing and comparison to BNP. Eur J Heart Fail 6:289-293

18. Melzi d'Eril G, Tagnochetti T, Nauti A et al (2003) Biological Variation of N-Terminal Pro-Brain Natriuretic Peptide in Healthy Individuals. Clin Chem 49:1554-1555

19. Emdin M, Passino C, Prontera C et al (2007) Comparison of Brain Natriuretic Peptide (BNP) and Amino-Terminal ProBNP for Early Diagnosis of Heart Failure. Clin Chem 53:289-1297

20. Zhu BL, Ishikawa T, Michiue $T$ et al (2007) Postmortem pericardial natriuretic peptides as markers of cardiac function in medicolegal autopsies. Int J Legal Med 121:28-35

21. Mueller T, Gegenhuber A, Dieplinger B, Poelz W, Haltmayer M (2004) Long-term stability of endogenous B-type natriuretic peptide (BNP) and amino terminal proBNP (NT-proBNP) in frozen plasma samples. Clin Chem Lab Med 42:942-944

22. Gill D, Seidler T, Troughton RW et al (2004) Vigorous response in plasma N-terminal pro-brain natriuretic peptide (NT-BNP) to acute myocardial infarction. Clin Sci 106:135-139

23. Kragelund C, Gronning B, Kober L, Hildebrandt P, Steffensen R (2005) N-terminal pro-B-type natriuretic peptide and long-term mortality in stable coronary heart disease.[see comment]. N Engl J Med 352:666-675

24. Nowatzke WL, Cole TG (2003) Stability of N-Terminal Pro-Brain Natriuretic Peptide after Storage Frozen for One Year and after Multiple Freeze-Thaw Cycles. Clin Chem 49:1560-1562

25. Salzmann J, Flitcroft D, Bunce C, Gordon D, Wormald R, Migdal C (1998) Brain natriuretic peptide: identification of a second natriuretic peptide in human aqueous humour. $\mathrm{Br} \mathrm{J}$ Ophthalmol 82:830-834

26. Wolfensberger TJ, Holz FG, Ationu A, Carter ND, Bird AC (1994) Natriuretic peptides and their receptors in human neural retina and retinal pigment epithelium. German J Ophthalmol $3: 248-252$

27. Pfister R, Schneider CA (2004) Natriuretic peptides BNP and NTpro-BNP: established laboratory markers in clinical practice or just perspectives? Clin Chim Acta 349:25-38

28. Goetze JP, Gore A, Moller CH, Steinbruchel DA, Rehfeld JF, Nielsen LB (2004) Acute myocardial hypoxia increases BNP gene expression. FASEB J 18:1928-1930 03-1336fje

29. Clerico A, Emdin M (2004) Diagnostic accuracy and prognostic relevance of the measurement of cardiac natriuretic peptides: a review. Clin Chem 50:33-50

30. Corteville DCM, Bibbins-Domingo K, Wu AHB, Ali S, Schiller NB, Whooley MA (2007) N-terminal pro-b-type natriuretic peptide as a diagnostic test for ventricular dysfunction in patients with coronary disease: data from the heart and soul study. Arch Intern Med 167:483-489

31. Jarolim P (2006) Serum biomarkers for heart failure. Cardiovas Pathol 15:144-149

32. Marz W, Tiran B, Seelhorst U, Wellnitz B, Bauersachs J, Winkelmann BR, Boehm BO (2007) N-terminal pro-B-type natriuretic peptide predicts total and cardiovascular mortality in individuals with or without stable coronary artery disease: the Ludwigshafen risk and cardiovascular health study. Clin Chem 53:1075-1083 